\title{
THE SEISMIC STRUCTURE OF THE SUN FROM GONG
}

\author{
E. ANDERSON ${ }^{1}$, H.M. ANTIA ${ }^{2}$, S. BASU ${ }^{3}$, B. CHABOYER ${ }^{4}$, \\ S.M. CHITRE ${ }^{2}$, J. CHRISTENSEN-DALSGAARD ${ }^{3,5}$, \\ A. EFF-DARWICH ${ }^{6}$, J.R. ELLIOTT ${ }^{7}$, P.M. GILES ${ }^{8}$, \\ D.O. GOUGH ${ }^{9,10}$, J.A. GUZIK ${ }^{11}$, J.W. HARVEY ${ }^{1}$, F. HILL ${ }^{1}$, \\ J.W. LEIBACHER ${ }^{1}$, A.G. KOSOVICHEV ${ }^{8}$, \\ M.J.P.F.G. MONTEIRO ${ }^{12}$, O. RICHARD ${ }^{13}$, T. SEKII $^{9}$, \\ H. SHIBAHASHI ${ }^{14}$, M. TAKATA $^{14}$, M.J. THOMPSON ${ }^{15}$ \\ J. TOOMRE ${ }^{7}$, S. VAUCLAIR ${ }^{13}$ AND S.V. VORONTSOV ${ }^{15}$ \\ ${ }^{1}$ National Solar Observatory, Tucson, Arizona, USA \\ ${ }^{2}$ Tata Institute of Fundamental Research, Bombay, India \\ ${ }^{3}$ Theoretical Astrophysics Center, Danish National Research \\ Foundation \\ ${ }^{4}$ Canadian Institute for Theoretical Astrophysics, Toronto, \\ Canada \\ ${ }^{5}$ Institute of Physics and Astronomy, Aarhus University, \\ Denmark \\ ${ }^{6}$ Center for Astrophysics, Cambridge, Massachusetts, USA \\ ${ }^{7}$ JILA, Boulder, Colorado, USA \\ ${ }^{8}$ HEPL, Stanford University, California, USA \\ ${ }^{9}$ Institute of Astronomy, University of Cambridge, UK \\ ${ }^{10}$ DAMTP, University of Cambridge, UK \\ ${ }^{11}$ Los Alamos National Laboratory, New Mexico, USA \\ ${ }^{12}$ University of Porto, Portugal \\ ${ }^{13}$ Observatoire Midi-Pyrénées, Toulouse, France \\ ${ }^{14}$ University of Tokyo, Japan \\ ${ }^{15}$ Queen Mary $\&$ Westfield College, University of London, UK
}

\section{Introduction}

This paper is an interim report of our inferences about the hydrostatic structure of the Sun, following the first report of the GONG team in Science (Gough et al., 1996). That work confirms that the spherically averaged structure of the Sun is more or less in agreement with current standard solar models. However, there remain some significant deviations which we 
regard as important clues to the existence of dynamical phenomena which are not taken into account in standard solar modelling.

\section{Frequency data}

The acoustic spectrum, as obtained from the GONG data, is described by Hill et al. (1996). The data used for the inversions reported here for the spherically averaged structure of the Sun were the frequencies of modes with degrees $l$ between 0 and 150. Only frequencies between 1.5 and 3.0 $\mathrm{mHz}$ were used for the inversions. Data from GONG months $1,2,4$ and 5 were used. (GONG months are contiguous intervals of 36 days, the first being centred about 24 May, 1995.) For the study of asphericity, the even component of degeneracy splitting was determined by fitting the formula $\nu_{n l m}=\nu_{n l}+L \Sigma_{i} a_{i} P_{i}(m / L)$ to the $m-\nu$ power spectra from GONG months 1 and 2 .

\section{The spherically averaged structure}

The spherically averaged structure of the Sun is inferred by comparing the multiplet oscillation frequencies (uniformly weighted averages of the components with like order $n$ and degree $l$, but with different azimuthal degrees $m$ ) of the Sun with degenerate eigenfrequencies of a (spherically symmetrical) standard solar model. A variety of inversion procedures have been carried out. Most commonly the frequency differences between Sun and model are expressed as averages of the differences of two state variables in the Sun, such as $c^{2}=\gamma p / \rho$ and $\rho$, or $\rho$ and $\gamma$, where $p, \rho$ and $\gamma$ are respectively pressure, density and the first adiabatic exponent $(\partial \ln p / \partial \ln \rho)_{s}$. Those averages are then combined linearly to produce more localized averages of a desired variable, using either one of the optimally localized averaging procedures or a regularized least-squares data-fitting algorithm.

The reliability of the inversion procedures has been tested using artificial data computed from a theoretical model of the Sun whose structure was unknown to the inverters. Some of the results of those tests are summarized in the contributed proceedings of this symposium (Antia et al., 1997). On the whole the results of the inversions agreed with the proxy Sun to within about a quoted standard error, except very near the surface and very near the centre of the Sun.

\section{The solar sound-speed}

We express the inferences as differences between averages of the sound speed in the Sun and corresponding sound-speed averages of a reference standard theoretical solar model. In all cases the reference model is that 
of Christensen-Dalsgaard et al. (1996) in which gravitational settling of helium and heavy elements is taken into account, but in which no other mechanism for redistribution of helium in the radiative zone is included. The model has an initial uniform heavy-element to hydrogen abundance ratio $Z / X=0.02768$, an initial helium abundance $Y=0.2713$, and an age of $4.60 \times 10^{9} \mathrm{y}$.

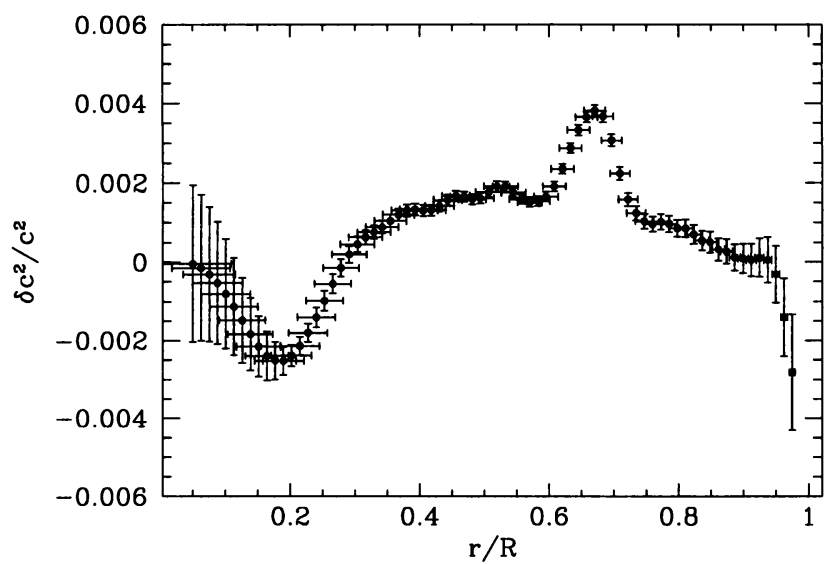

Figure 1. Relative difference $\delta c^{2} / c^{2}$ between the square of the sound speed $c$ in the Sun and that in the standard reference theoretical model. The horizontal bars represent the widths of the averaging kernels; the vertical bars represent standard errors.

A sample inversion is illustrated in Figure 1. Because there is reason to be wary of the accuracy of the higher solar frequencies, which have greater line widths, and also the lower frequencies, for which the signal-to-noise ratio is relatively low, modes in somewhat different frequency ranges have been used.

There are three prominent features of the inversions illustrated in Figure 1 which are common to all the inversions and which merit attention:

1. there is a sharp peak in the sound-speed difference immediately beneath the base of the convection zone;

2. the sound speed in the radiative envelope, outside the core, is somewhat greater in the Sun than it is in the model;

3. except possibly in the very central region, the sound speed in the energy generating core of the Sun is lower than in the model.

We suspect that the differences in the outer layers of the convection zone may be a product of inaccurate modelling of the surface layers of the Sun, and we refrain from discussing them.

The peak in $\delta c^{2} / c^{2}$ immediately beneath the convection zone is hardly a surprising feature of the inversions given that the only mechanism in the reference model for transporting material, aside from convection, is 
gravitational settling. In reality, motion beneath the convection zone, such as might be induced by convective penetration, is likely to cause significant mixing. In particular, helioseismic inversion has shown the presence of a thin region of rotational shear immediately beneath the convection zone (e.g. Thompson et al., 1996; Korzennik et al., these proceedings; Sekii, these proceedings), which has been dubbed the tachocline; here there must be a circulatory flow driven by the pressure imbalance on level surfaces (e.g. Spiegel and Zahn, 1992), which must modify the compositional gradients that result from settling. The advection of excess helium and heavy elements back into the convection zone, and the consequent replenishment of hydrogen from the convection zone into the shear layer, must surely raise the sound speed. Of course, there will be another contribution to the sound-speed adjustment due to a change in temperature resulting from the opacity change. But, since that effect is primarily a change to the gradient of temperature, rather than its absolute value, the local influence on the sound speed is likely to be smaller. Detailed comparison of the distribution of both sound speed and angular velocity will, it is to be hoped, enable theories of the shear layer to be calibrated (Kosovichev, 1996; Gough and Sekii, 1997).

A second contributor to the peak in $\delta c^{2} / c^{2}$ immediately beneath the convection zone could have been substantial mass loss (about $0.1 M_{\odot}$ ) by the Sun. If mass loss were to have taken place, material that is now immediately beneath the convection zone would previously have been relatively deeper and more compressed. Under those conditions the augmentation of the abundances of helium and heavy elements would have been less than it is in the standard model, thereby contributing to an augmentation in $c^{2}$. The solar sound speed is compared with a model suffering mass loss in Figure 2. Notice that the peak in $\delta c^{2} / c^{2}$ is hardly present. However, the oscillation frequencies appear not to be reproduced perfectly.

There are many potential causes for the remaining sound-speed excess in the bulk of the radiative zone: $0.3 \lesssim r / R \lesssim 0.65$, and it is not unlikely that the actual cause is a combination of several of them. Some could act locally, such as errors in the value of $\gamma$ which influence $c^{2}$ directly without having a direct influence on the structure of the radiative zone in the model. Others act more globally, such as errors in opacity, and errors in the equation of state that influence quantities other than $\gamma$. (Such errors must surely be present if there is an error in $\gamma$.) Their influence is spread partly by the global nature of the differential equations that determine the overall stratification, and partly by the changes in initial conditions demanded by the calibration of the radius and luminosity of the present Sun. ChristensenDalsgaard (1996) has discussed the influence on model structure of a wide variety of changes to many of the structure variables. 


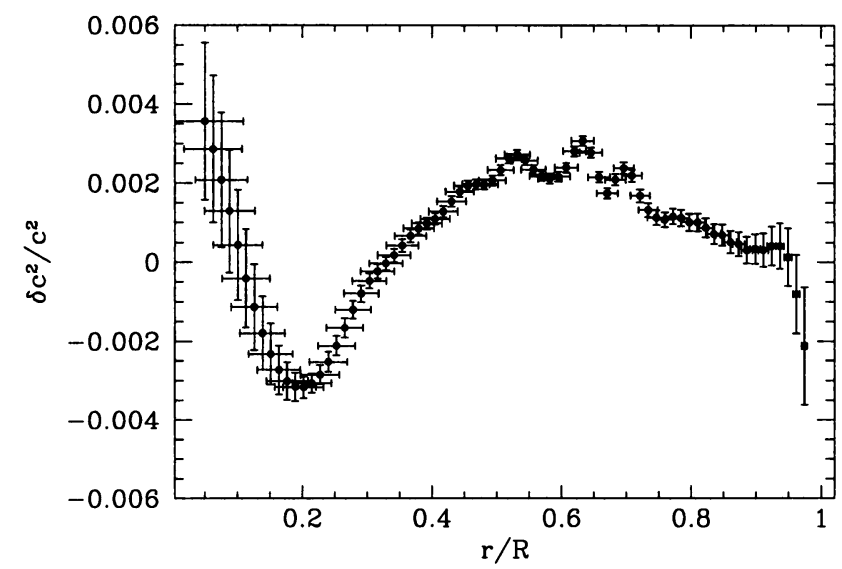

Figure 2. Relative difference $\delta c^{2} / c^{2}$ between the square of the sound speed $c$ in the Sun and that in a model computed by Guzik and Cox (1995) that has suffered mass loss.

The excess sound speed in the outer part of the core $(0.1 \lesssim r / R \lesssim 0.3)$ of the reference model is particularly interesting because it is a symptom of erroneous modelling of the region in which the nuclear reactions are taking place. The relevance of that to the solar neutrino problem is obvious. Knowledge of this discrepancy is not new, though the details have been in question, and perhaps should be regarded as still being in some doubt. Although the discrepancy is substantially greater than the formal standard errors that reflect the random errors in the data, it must be borne in mind that it arises from relatively small contributions to the oscillation frequencies. We must be aware that it might therefore be the product of some small systematic error in the data analysis, either in the procedure for converting raw data to oscillation frequencies, or in the assumptions upon which the frequency inversion schemes rely. How the discrepancy arises has not yet been determined, but we remind the reader that one possibility is that, as in the case of the rotational shear layer at the base of the convection zone, it could be a consequence of macroscopic material motion redistributing the helium that is produced by the nuclear reactions. If that were to be so, then one would expect the decline in the mean sound-speed difference in the outer layers of the core to be associated with a corresponding increase at the centre, caused by the hydrogen-rich material that has been brought from greater radii to displace the central core. Such a possibility is hinted at by the GONG data, but it is not demonstrated. We note also that an error in the assumed age of the Sun can also produce a deviation in the core and the radiative envelope not wholly unlike that implied by the inversions (Gough and Novotny, 1990), but it does not account for the entire discrepancy. 


\section{Density distribution}

An example of an inversion for density is illustrated in Figure 3. The relative differences are greater than for sound speed, but can be interpreted in a consistent fashion. The general rise of $\delta \rho / \rho$ with radius indicates that the decline in the actual density in the Sun is somewhat more gradual than it is in the reference model; the Sun is less centrally condensed than the model. In particular, the especially sharp gradient in $\delta \rho / \rho$ immediately beneath the convection zone, which indicates a localized flattening of the density gradient, could be a direct consequence of the tendency of the tachocline circulation to homogenize the chemical composition. A reduction in the radial variation of the spherically averaged helium abundance in the core might also account for the similar steep gradient of $\delta \rho / \rho$ inwards of $r / R \simeq$ 0.15 .

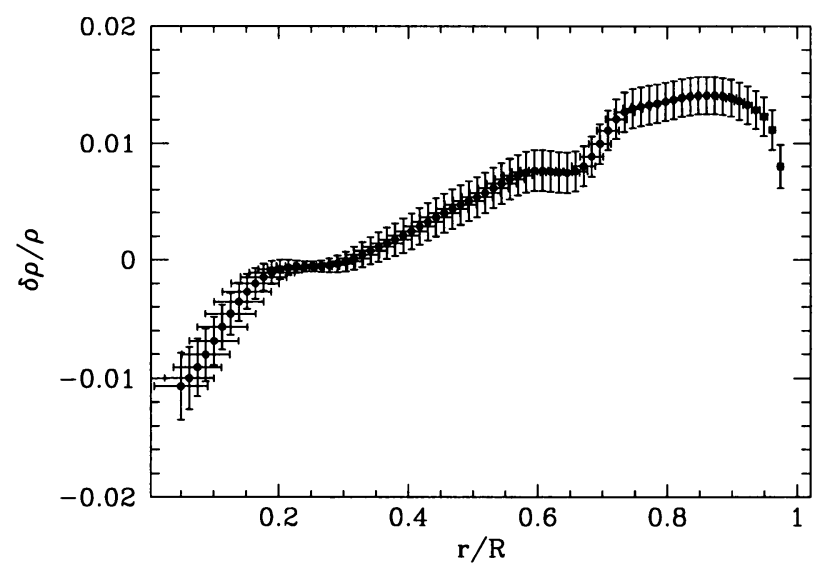

Figure 3. Relative difference $\delta \rho / \rho$ between the density $\rho$ in the Sun and that in the standard reference theoretical model.

\section{Asphericity}

One cannot distinguish from frequency data alone between the possible aspherical mechanisms that might be responsible for the even component of degeneracy splitting (Zweibel and Gough, 1995). We therefore represent the asphericity as a, possibly fictitious, variation of the wave propagation speed.

The even splitting coefficients $a_{i}$ vary with frequency in approximately the same manner as does the inverse of the modal inertia. This is consistent with the findings of Woodard and Libbrecht (1993). Therefore most of the contribution to the even component of degeneracy splitting must be 
confined to the outer layers of the star, near and above the upper turning points of the modes.

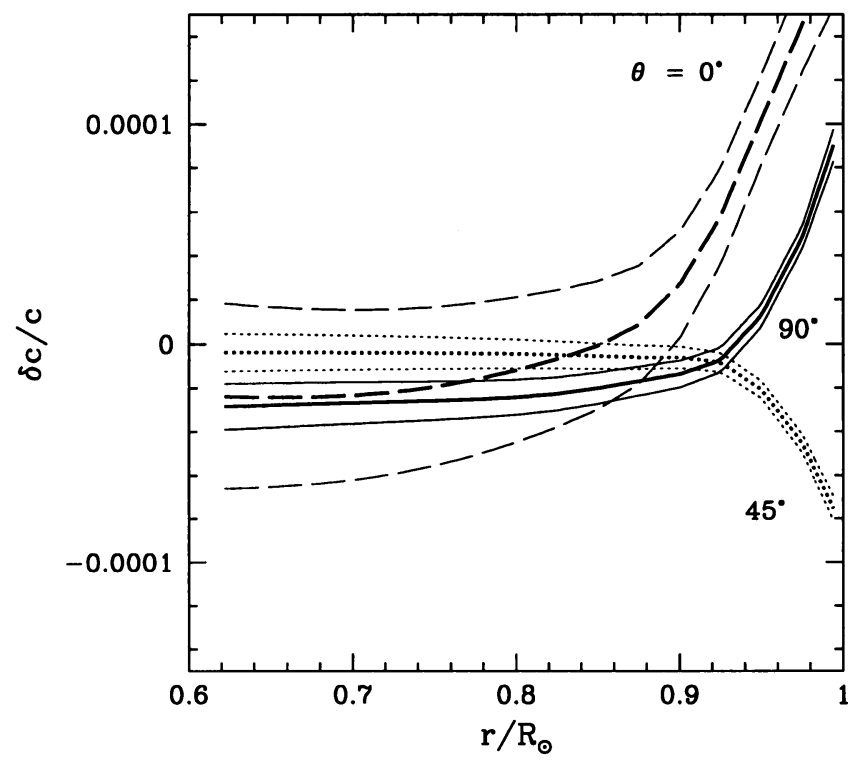

Figure 4. Relative wave-propagation-speed deviation from the spherical average, plotted against radius, at the equator $\left(\theta=90^{\circ}\right)$, at latitude $45^{\circ}$ and at the poles: thick lines represent expectations of the averages and the thin lines \pm one standard deviation from them.

Figure 4 illustrates the wave-speed deviation from the spherical average at three values of latitude. These deviations are positive at high and low latitudes, and negative at midlatitudes. That is in qualitative agreement with the findings of Woodard and Libbrecht for 1986, which was at a similar epoch in the solar cycle. We expect, as the sunspot cycle progresses, that this behaviour will reverse once there is substantial midlatitude magnetic activity.

\section{Thermal stratification at the base of the convection zone}

The meridional velocities induced by baroclinicity in the tachocline (Spiegel and Zahn, 1992; Gough and Sekii, 1997) are too small to perturb significantly the radiative heat flux. Consequently, the issue of the extent of the convective overshoot - by which we mean motion beneath the level at which the stratification is neutrally stable to convection and which is driven by pressure gradients induced by buoyancy forces within the convection zone - may be studied separately. Our interest is in the extent of the motion 
that is sufficiently vigorous to modify the thermal stratification, bringing it closer to being adiabatic.

Simple models of the overshoot region (e.g. Zahn 1991) suggest the existence of a nearly adiabatically stratified overshoot layer beneath which there is a rapid transition of the temperature gradient almost to the value it would have had in the absence of overshoot. Such a transition would induce contributions to the frequencies of the modes that are oscillatory with respect to frequency. To identify a possible oscillatory contribution we have considered fourth differences $\delta_{4} \nu$ of the frequencies at fixed $l$ (see Basu, 1997, for details). From solar models with varying extents of overshoot we have found that the amplitude of the oscillation in $\delta_{4} \nu$ increases monotonically with the extent of overshoot (Christensen-Dalsgaard et al., 1995). By comparing the solar value of $\delta_{4} \nu$, about $0.8 \mu \mathrm{Hz}$, with the model sequence, we conclude that any overshoot that might be present extends no more than about 0.05 pressure scale heights beneath the base of the convection zone.

Finally we point out that transport of material and angular momentum by gravity and inertial waves generated in the lower boundary layer of the convection zone and in the tachocline also play a role in influencing the sound-speed and angular-velocity variation. All the dynamical processes in this region control not only the $\mathrm{H}-\mathrm{He}$ abundance ratio but also the abundances of the light elements $\mathrm{Li}$ and $\mathrm{Be}$ in the convection zone.

\section{References}

Antia, H.M. et al. (1997) Sounding solar and stellar interiors, (ed. J. Provost and F-X. Schmider, Observatoire de la Côte d'Azur) in press

Basu, S. (1997) Mon. Not. R. Astron. Soc., in press

Christensen-Dalsgaard, J. (1996) in Proc. VI IAC Winter School: The structure of the Sun, (eds T. Roca Cortés \& F. Sánchez, Cambridge University Press), p. 47

Christensen-Dalsgaard, J., Monteiro, M.J.P.F.G. and Thompson, M.J. (1995) Mon. Not. R. astron. Soc., 276, 283

Christensen-Dalsgaard, J. et al. (1996) Science, 272, 1286

Gough, D.O. and Novotny, E. (1990) Solar Phys., 128, 143

Gough, D.O. et al. (1996) Science, 272, 1296

Gough, D.O. and Sekii, T. (1997) Sounding solar and stellar interiors, (ed. J. Provost and F-X. Schmider, Observatoire de la Côte d'Azur) in press

Guzik, J. and Cox, A.N. (1995) Astrophys. J., 448, 905

Hill, F. et al. (1996) Science, 272, 1292

Kosovichev, A.G. (1996) Astrophys. J., 469, L61

Spiegel, E.A. and Zahn, J-P. (1992) Astron. Astrophys., 265, 106

Thompson, M.J et al. (1996) Science, 272, 1300

Woodard, M.F. and Libbrecht, K.G. (1993) Astrophys. J., 402, L77

Zahn, J.-P. (1991) Astron. Astrophys., 252, 179

Zweibel, E.G. and Gough, D.O. (1995) Proc. Fourth SOHO Workshop: Helioseismology, (ed. J.T. Hoeksema, V. Domingo, B. Fleck and B. Battrick, European Space Agency SP-376, Noordwijk), vol 2, 73 